\title{
Serotonin and dopamine modulate aging in response to food perception and availability
}

2

3

4

5

6

\section{Authors:}

Hillary A. Miller ${ }^{1}$, Shijiao Huang ${ }^{2} *$, Megan L. Schaller ${ }^{2}$, Elizabeth S. Dean ${ }^{2}$, Angela M. Tuckowski ${ }^{1}$, Allyson S. Munneke ${ }^{1}$, Safa Beydoun ${ }^{2}$, Scott D. Pletcher ${ }^{2}$, and Scott F. Leiser ${ }^{2,3 \#}$

\section{Affiliations:}

1. Cellular and Molecular Biology Program, University of Michigan, Ann Arbor, MI 48109, USA.

2. Molecular \& Integrative Physiology Department, University of Michigan, Ann Arbor, MI 48109

3. Department of Internal Medicine, University of Michigan, Ann Arbor, MI 48109, USA.

* Both authors contributed equally to this work.

\# Corresponding author, Scott F. Leiser, leiser@umich.edu

\section{Keywords:}

C. elegans; D. melanogaster; mammalian cells; lifespan; aging; mianserin; thioridazine; serotonin; dopamine; cell-nonautonomous; flavin containing monooxygenase; fmo-2; dietary restriction; nervous system 


\section{Abstract}

22 An organism's ability to perceive and respond to changes in its environment is crucial for its

23 health and survival. Here we reveal how the most well-studied longevity intervention, dietary

24 restriction (DR), acts in-part through a cell non-autonomous signaling pathway that is inhibited

25 by the perception of attractive smells. Using an intestinal reporter for a key gene induced by DR

26 but suppressed by attractive smells, we identify three compounds that block food perception in

27 C. elegans, thereby increasing longevity as DR mimetics. These compounds clearly implicate

28 serotonin and dopamine in limiting lifespan in response to food perception. We further identify

29 an enteric neuron in this pathway that signals through the serotonin receptor $5-\mathrm{HT} 1 \mathrm{~A} / \mathrm{ser}-4$ and

30 dopamine receptor DRD2/dop-3. Aspects of this pathway are conserved in D. melanogaster and

31 mammalian cells. Thus, blocking food perception through antagonism of serotonin or dopamine

32 receptors is a plausible approach to mimic the benefits of dietary restriction.

\section{Main}

35 Rapid advances in aging research have identified several conserved signaling pathways that

36 influence aging in organisms across taxa ${ }^{1}$. Recent work shows that many of these "longevity

37 pathways" act through cell non-autonomous signaling mechanisms ${ }^{2,3}$. These pathways utilize

38 sensory cells - frequently neurons - to signal to peripheral tissues and promote survival during

39 the presence of external stress. Importantly, this neuronal activation of stress response pathways,

40 through either genetic modification or exposure to environmental stress, is often sufficient to

41 improve health and longevity. Despite mounting evidence that neuronal signaling can influence

42 multiple longevity pathways, less is known about which specific cells and molecules propagate

43 these signals. 
Biogenic amines are among the most well-studied and conserved neuronal signaling

45 molecules ${ }^{4,5}$. Specifically, serotonin and dopamine play well-defined roles in behavior and

46 physiology. However, their role in aging is less well understood. Several recent studies implicate

47 serotonin, but not dopamine, as an important signal in multiple C. elegans longevity pathways

48 including the response to heat shock and hypoxia ${ }^{6,7}$. Dopaminergic signaling is associated with

49 physical activity in humans and loss of this signaling decreases lifespan in mice ${ }^{8}$ and blocks

50 lifespan extension in nematodes ${ }^{9}$. Serotonin and dopamine levels both decrease with age across

51 species $^{10,11}$, consistent with these signaling pathways promoting healthy aging. Despite rigorous

52 study and clinical use of drugs that modify serotonin and dopamine signaling, our understanding

53 of their complex actions and potential interaction is far from complete.

54 Dietary restriction (DR) is the most well-studied and consistent intervention known to

55 improve health and longevity in organisms ranging from single-celled yeast to primates ${ }^{12}$. DR

56 leads to improved cell survival and stress resistance, complex intracellular signaling events,

57 metabolic changes, and increased activity in multiple organisms. Nematode flavin-containing

58 monooxygenase-2 (fmo-2) is necessary and sufficient to increase health and longevity

59 downstream of DR. FMOs are highly conserved proteins that are also induced in multiple

60 mammalian models with increased lifespan ${ }^{13,14}$. Having previously identified a role for $f m o-2$ in

61 aging, we wondered whether DR cell non-autonomously regulates fmo-2 induction and whether

62 perception of food through biogenic amines could be involved in the subsequent signaling

63 pathway.

64 


\section{Results}

66 Attractant food perception represses fmo-2 to limit longevity.

67 We developed an integrated single-copy $m$ Cherry reporter driven by the fmo- 2 promoter to

68 measure $f m o-2$ induction. The reporter is primarily expressed in the intestine and responds to

69 stimuli previously reported to induce fmo-2, including DR. As an intestinal protein ${ }^{15}$, we

70 expected that fmo- 2 would likely be induced cell autonomously by the change in nutrient intake

71 under DR. To test this hypothesis, we asked whether the perception of food smell by worms in

72 the absence of eating can abrogate the induction of fmo-2. Using a "sandwich plate" assay as

73 described in Figure 1A, we were surprised to find a significant reduction in fmo-2 induction

74 when worms could smell but not eat food (Figure 1B-C). This reduction is consistent with a

75 model in which increased fmo-2 mediates the increase in longevity from DR, as food smell

76 completely abrogates this lifespan extension (Figure 1D, lifespan statistics in Table S1) ${ }^{16}$. We

77 also find that active bacterial metabolism is required to abrogate fmo-2 induction, as the "smell"

78 of bacteria metabolically killed with $0.5 \%$ paraformaldehyde does not prevent DR from inducing

79 fmo-2 expression (Figure S1A-B). Since intestinal cells are not known to perceive external

80 environmental cues such as smell, these results suggest that fmo-2 expression is suppressed when

81 food is present through cell non-autonomous signaling.

83 We next wondered what types of odorant compounds worms sense in this pathway. Bacteria are

84 known to secrete hundreds of volatile compounds that are classified in three categories based on

85 how they promote chemotaxis: attractants, repellants, and neutral compounds ${ }^{17-19}$. We tested

86 whether exposure to any volatile compound secreted from bacteria is sufficient to block the

87 lifespan-promoting effects of DR or whether compounds identified as attractants and repellants 
88 oppositely regulate fmo-2 induction. Using compounds derived from studies of the E. coli strain

89 HB101 in a range of concentrations (Table S2), we find that attractants are more likely to

90 suppress DR-mediated induction of fmo-2 (Figure 1E-F) whereas neutral and repellant

91 compounds can induce fmo-2 under fed conditions (Figure S1C-H). We also find that many

92 compounds suppress fmo-2 expression, consistent with the hypothesis that this pathway is not

93 acting through a single receptor (Figure 1G, all results in Figure S2A-Z). These results support a

94 model in which perception of attractive smells secreted by E. coli abrogates the induction of the

95 pro-longevity gene $f m o-2$. This is consistent with these smells preventing the lifespan-promoting

96 effects of DR, possibly through a neural response to external stimuli that leads to physiological

97 changes in peripheral tissues. 
A

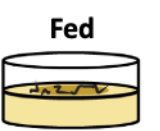

B

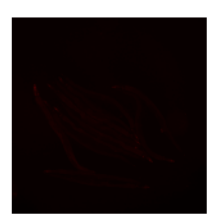

Fed

E

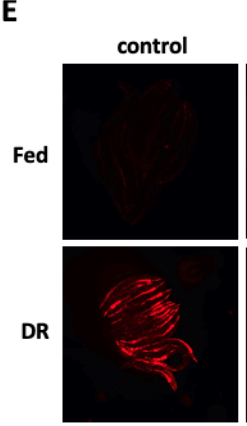

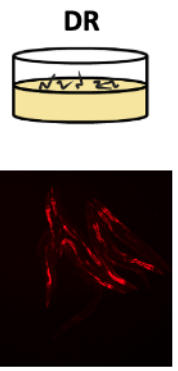

DR
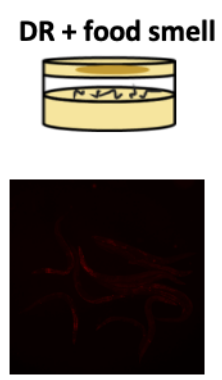

DR + food smell
+ food smell

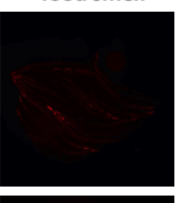

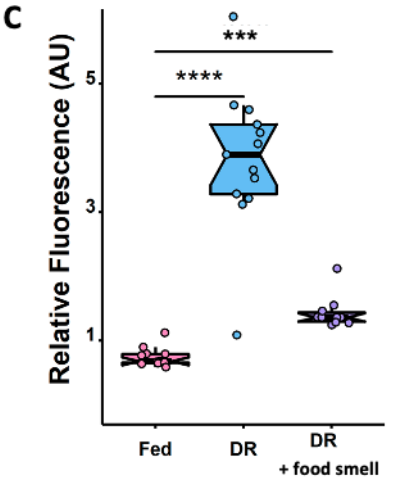

$\mathbf{F}$
78
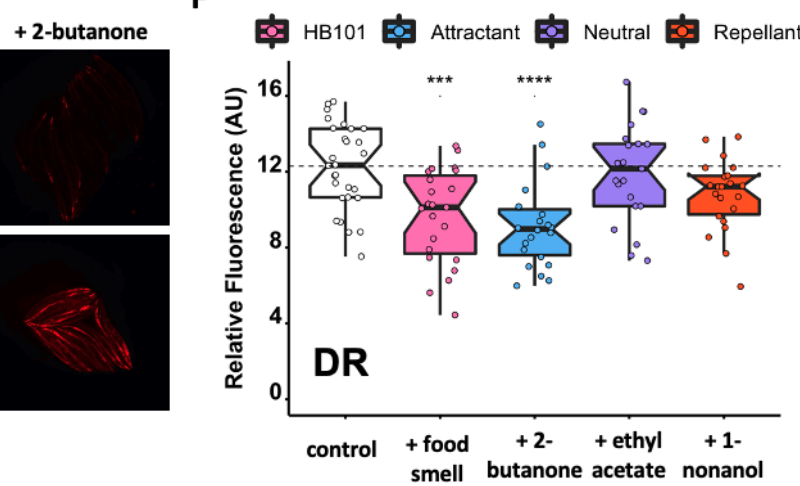

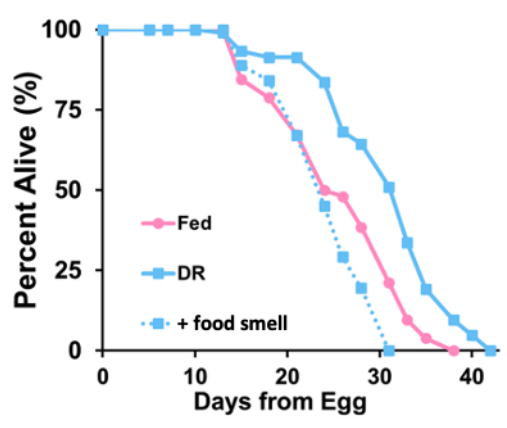

G

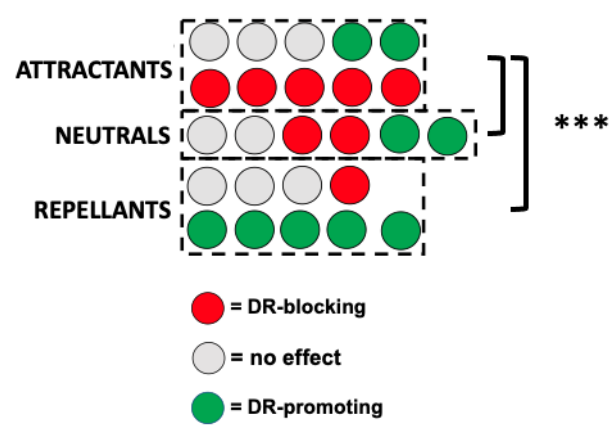

Figure 1. Attractive food smell blocks dietary restriction-mediated fmo-2 induction and longevity. Diagram of

"smell plates" (A). Images (B) and quantification (C) of individual fmo-2p::mCherry worms on fed (pink), DR (blue) and DR + food smell (OP50) (purple). Survival curves (D) of N2 (WT) animals fed (pink) or DR (blue) under normal conditions (solid lines) or subjected to the smell of bacteria (dotted lines). Images (E) and quantification (F) of individual fmo-2p::mCherry worms on DR plates exposed to food smell (HB101) (pink) or attractive (2-butanone in blue), neutral (ethyl acetate in purple), or repellant (1-nonanol in orange) odorants. (G) Summary of the effects of 26 odorants on fmo-2 induction during DR. *** denotes $\mathrm{P}<.001$, **** denotes $\mathrm{P}<.0001$ when compared to fed (Tukey's HSD) \#\#\# denotes $\mathrm{P}<$ 0.001 when compared to neutrals (ANOVA). 


\section{Serotonin and dopamine antagonists induce fmo-2 to mimic DR longevity.}

108 Biogenic amines can regulate pro-longevity pathways and are involved in behavioral changes in

109 response to food ${ }^{6,7,20-22}$. We next asked whether neurotransmitters are involved in the fmo-2-

110 mediated food perception pathway. Using a targeted approach focusing on neurotransmitters and

111 their antagonists, we tested for compounds sufficient to prevent the abrogation of fmo-2

112 induction in the presence of food smell (Figure S3A-D). The biogenic amine neurotransmitter

113 antagonists mianserin (for serotonin) and thioridazine and trifluoperazine (for dopamine)

114 consistently and significantly restore fmo-2 induction to DR levels in the presence of food smell

115 (Figure 2A-C, Figure S3E-F). Mianserin is a tetracycline serotonin antagonist that is thought to

116 competitively bind to specific serotonergic G protein-coupled receptors (GPCRs) ${ }^{23}$ while

117 thioridazine and trifluoperazine's mechanism of action involves blocking dopamine receptors ${ }^{24}$.

118 Importantly, while each compound induces fmo-2 to a different extent (Figure 2D, Figure

119 S3Gand S3I), when combined with DR, no antagonist further induced fmo-2, suggesting they act

120 in the same pathway (Figure 2E, Figure S3H-I). Diphenyleneiodonium chloride (DPI), an

121 inhibitor of NADPH oxidase, acts as a positive control, and further induces fmo- 2 when

122 combined with DR (Figure 2E). Because thioridazine and trifluoperazine act through similar

123 mechanisms and the effects of thioridazine were more consistent in our studies, we focused

124 further experiments on dopamine antagonism through thioridazine. Together, these results

125 support antagonism of serotonin or dopamine as partial mimetics of DR in their induction of

126 fmo-2.

127

128 To validate that the induction of fmo-2 through biogenic amine antagonism is beneficial for

129 longevity, we next asked whether these compounds extend lifespan. We find that both mianserin 
130 and thioridazine extend lifespan on agar plates in a dose-dependent manner (Figure 2F-G $)^{25}$.

131 Since we identified mianserin and thioridazine through their induction of $f m o-2$, and previously

132 found that fmo-2 is necessary for DR-mediated lifespan extension, we next asked whether fmo-2

133 was necessary for the beneficial longevity effects of mianserin or thioridazine. Our results show

134 that the fmo-2 loss of function completely blocks the lifespan effect of mianserin (Figure $\mathbf{2 H}$ )

135 and thioridazine (Figure 2I). Importantly, we also see that mianserin treatment combined with

136 DR does not further extend lifespan (Figure S3J). These results are consistent with these

137 compounds mimicking some aspects of DR-signaling, recapitulating part of the DR lifespan

138 extension effect. Collectively, this supports a model where DR induces fmo-2 because of

139 decreased biogenic amine signaling and establishes neuromodulators as a useful tool to decipher

140 where in the signaling pathway a cell, signal, or receptor plays a role in DR-mediated longevity. 
A
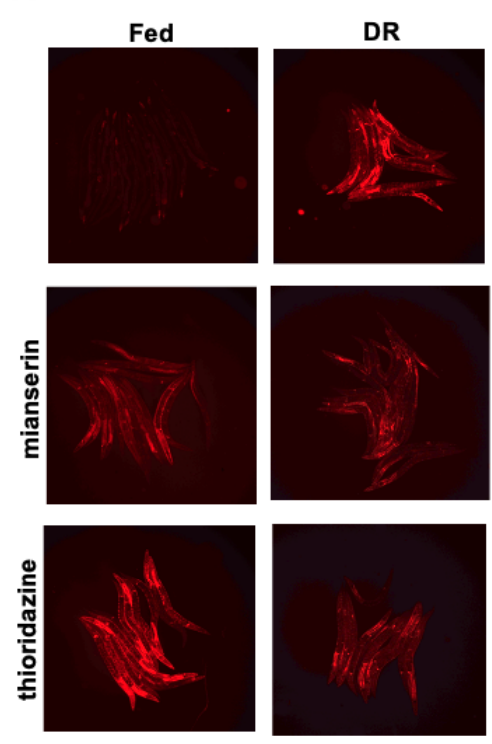

F

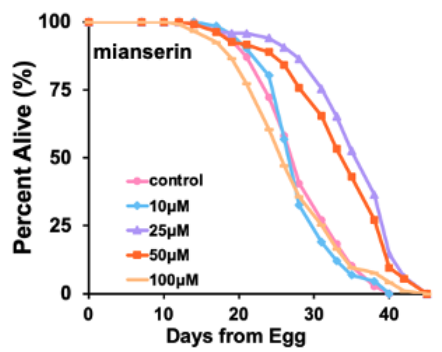

$D R+$ food smell
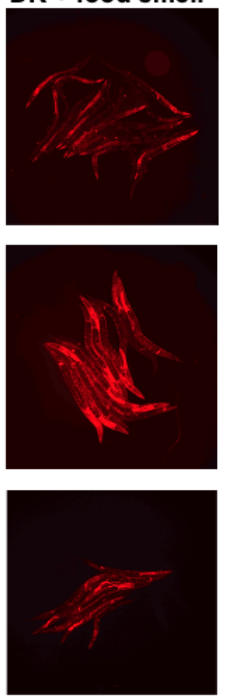

G

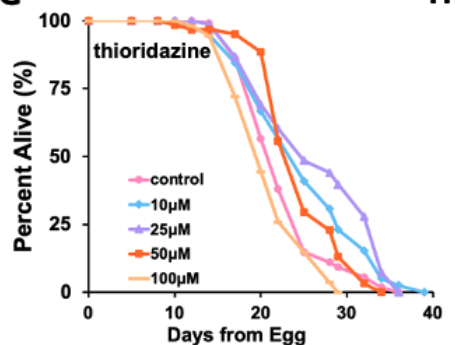

food smell

D

H
B
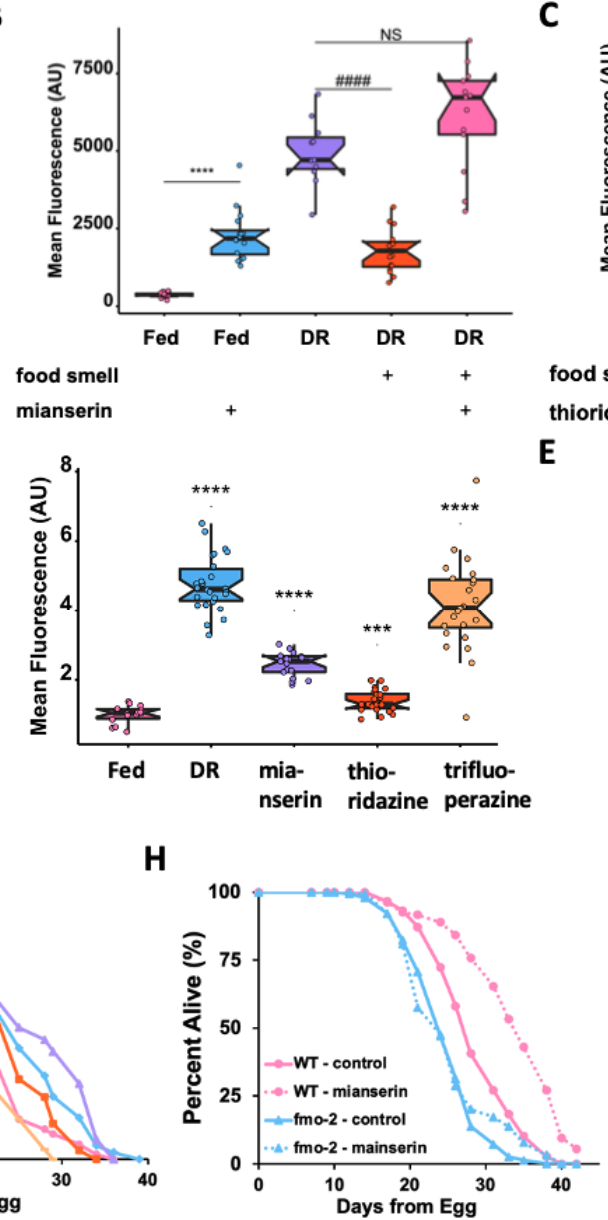

C

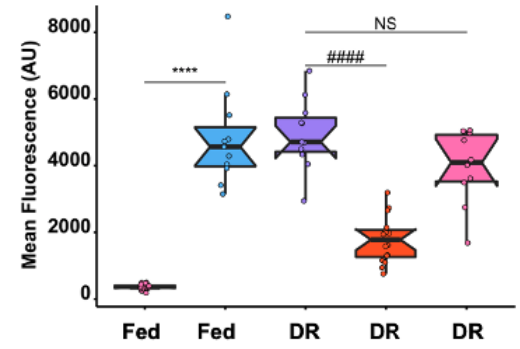

food smell

thioridazine

E

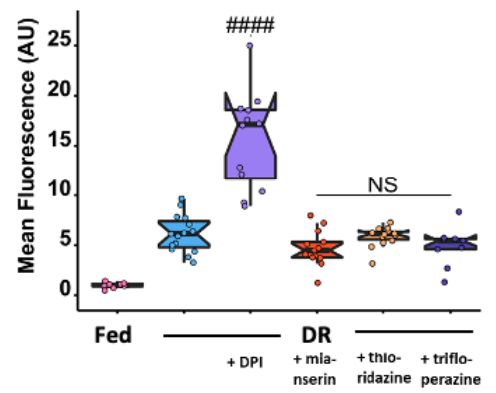

I

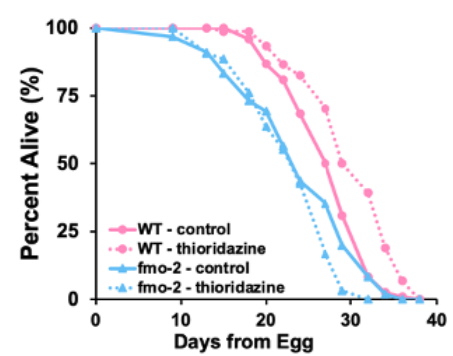

Figure 2. Serotonin and dopamine antagonists induce fmo-2 and extend lifespan. Images (A) and quantification of

3 fmo-2p::mCherry exposed $100 \mu \mathrm{M}$ of mianserin (B) or thioridazine (C) (blue) in combination with DR (orange) and food smell (pink) compared to DR alone (purple). Quantification (D) of fmo-2p::mCherry exposed to water (pink), DR (blue), $100 \mu \mathrm{M}$ mianserin (purple), thioridazine (orange), or trifluoperazine (yellow). Quantification (E) of fmo-2p::mCherry

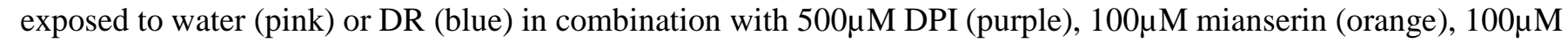
thioridazine (yellow), or $100 \mu \mathrm{M}$ trifluoperazine (dark purple). Survival curves (F) of N2 (WT) animals treated with $0 \mu \mathrm{M}$ (water; pink), $10 \mu \mathrm{M}$ (blue), $25 \mu \mathrm{M}$ (purple), $50 \mu \mathrm{M}$ (orange), or $100 \mu \mathrm{M}$ (yellow) mianserin. Survival curves (G) of WT animals treated with $0 \mu \mathrm{M}$ (water; pink), $10 \mu \mathrm{M}$ (blue), $25 \mu \mathrm{M}$ (purple), $50 \mu \mathrm{M}$ (orange), or $100 \mu \mathrm{M}$ (yellow) thioridazine.

Survival curves $(\mathbf{H})$ of WT animals (pink) and fmo-2 KO animals (blue) on water (solid lines) or 50 $\mu \mathrm{M}$ mianserin (dotted lines). Survival curves (I) of WT animals (pink) and fmo-2 KO animals (blue) on water (solid lines) or $25 \mu \mathrm{M}$ thioridazine (dotted lines). *** denotes $\mathrm{P}<.001, * * * *$ denotes $\mathrm{P}<.0001$ when compared to fed (Tukey’s HSD). \#\#\#\# denotes $\mathrm{P}<.0001$ when compared to DR (Tukey's HSD). 


\section{DR signaling acts through a pair of enteric neurons.}

155 Our initial results establish that antagonizing serotonin and dopamine signaling leads to

156 induction of the longevity promoting fmo-2 gene and rescue of the negative effects of food smell.

157 Based on this, we hypothesized that the relative lack of food smell during DR leads to increased

158 longevity through induction of intestinal fmo-2. Using this framework, we next sought to better

159 understand how the sensing of bacteria (or lack thereof) is communicated to intestinal cells

160 during DR. Our results, knocking down the synaptic vesicle exocytosis gene unc-13, support

161 short-range neurotransmitters as necessary for fmo-2 induction (Figure S4A-B).

162

163 In C. elegans, perception of the external environment is largely regulated by a specialized organ

164 known as the amphid. Since a previous report using a solid-liquid DR approach suggested a

165 pathway originating in the ASI amphid neurons, we first asked whether these cells are required

166 to modulate $f m o-2$ activity during $\mathrm{DR}^{26}$. We find that not only are the ASI neurons (as measured

167 by $d a f-3$ and $d a f-7$ RNAi) dispensable for food perception-mediated reduction in fmo-2

168 expression (Figure S4C-D), but proper formation of the amphid (daf-6) is also not required

169 (Figure S4E-F). This result is consistent with a non-canonical sensory neuron playing a role in

170 food perception-mediated fmo-2 suppression.

172 To better map this pathway, we next asked whether the biogenic amine serotonin is involved in

173 the DR-mediated longevity pathway, and if so, where. We tested whether knocking out serotonin

174 signaling would mimic the effects of DR. We subjected animals lacking $t p h-1$, the rate-limiting

175 enzyme necessary to produce serotonin, to DR and mianserin. $t p h-1$ animals are long-lived

176 compared to wild-type ${ }^{27}$ and not further extended by our DR protocol (Figure 3A) or mianserin 
177 treatment (Figure S5A). These data are supported by the abatement of fmo-2 induction on DR

178 (Figure 3B-C) and mianserin (Figure S5B-C) when animals are subjected to tph-1(RNAi). As

179 post-mitotic animals, C. elegans have a finite number of neurons with discrete connectivity and

180 functions. Three neuronal pairs normally express $t p h-1^{28}$. The hermaphrodite specific motor

181 neurons (HSN) are located along the ventral tail and regulate egg-laying ${ }^{29}$ whereas two head

182 neuron pairs, the amphid neurons with dual sensory endings (ADF) and the neurosecretory motor

183 (NSM) neurons, are involved in modifying behavioral states ${ }^{20,30,31}$. To investigate the potential

184 role of these neuron pairs, we utilized tph-1 cell-specific knockout and rescue strains and found

185 that tph-1 expression in NSM, but not the ADF, neurons (Figure S5D-E) is necessary (Figure

186 S5F) and sufficient (Figure 3D) to promote DR-mediated longevity. These results implicate the

187 NSM neurons as two of the primary neurons involved in reversing the effects of DR under food 188 smell.

190 Recent research posits that NSM neurons function similar to enteric neurons with neural

191 projections that directly communicate with the pharynx through a pair of acid-sensing ion

192 channels (ASICs), DEL-3 and DEL-7. Signaling through these channels informs the worm to

193 slow locomotion upon contact with food ${ }^{30}$. These data led us to wonder whether the longevity

194 effects of DR also require the ASICs channels to extend lifespan. We find that del-7 mutants

195 look phenotypically wild type in their induction of fmo-2 and lifespan extension, in either DR or

196 DR + food smell (Figure S6A-C). Interestingly, del-3 mutant worms show abrogated induction

197 of fmo-2 under DR, and did not diminish fmo-2 induction in response to the smell of food

198 (Figure 3E-F). These del-3 mutant animals still exhibit lifespan extension under DR, despite the 199 decreased induction of fmo-2, which is not abrogated by the smell of food (Figure 3G). 
200 Together, these data support a model whereby the enteric NSM neurons release serotonin in

201 response to food perception and the lack of this release extends longevity. In addition, the ASIC

202 DEL-3 plays a role in the NSM to both behaviorally ${ }^{30}$ and physiologically respond to food

203 perception signals. 
A

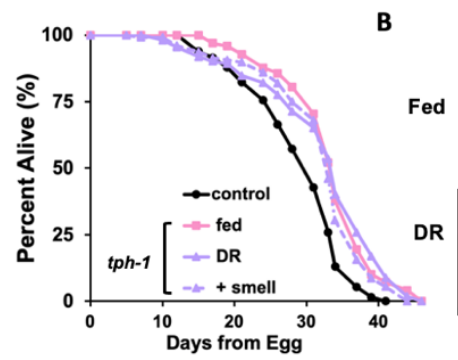

E

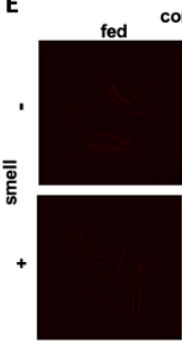

control

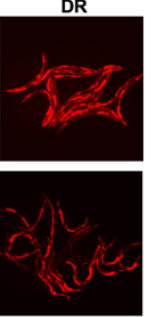

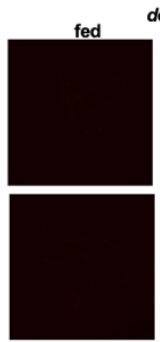
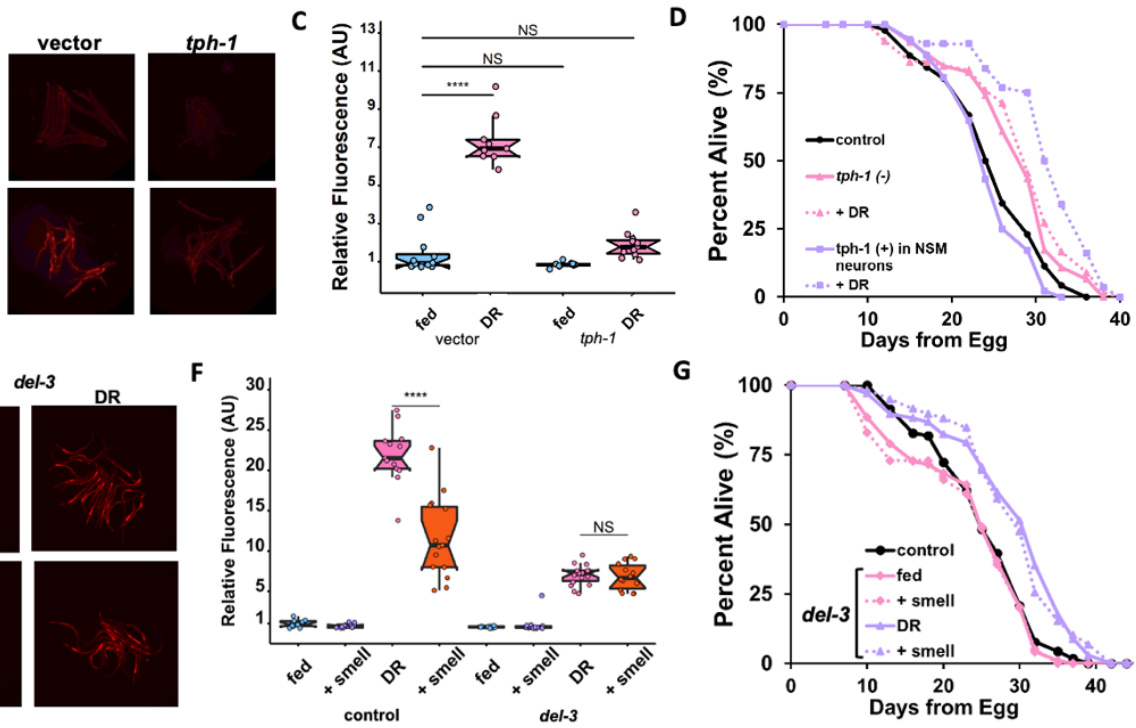

G

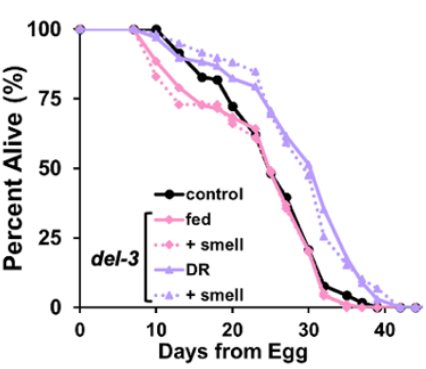

Figure 3. Food signals emanate from the NSM neurons. Survival curves (A) of WT animals (black) and tph-1 KO animals on fed (pink) and DR (purple) conditions exposed to food smell (dotted lines). Images (B) and quantification (C) of fmo-2p::mCherry exposed to tph-1 RNAi on fed (blue) or DR (pink). Survival curves (D) comparing control (black), tph-1 KO (pink), and tph-1 NSM-specific rescue (purple) animals on fed (solid line) and DR (dotted lines). Images (E) and quantification (F) of fmo-2p::mCherry

210 in a WT (control) and del-3 background on fed (blue) and DR (pink) exposed to food smell (purple and

211 orange, respectively). Survival curves of conditions comparing WT (black) to del-3 (G) on fed (pink) and

212 DR (purple) conditions in combination with food smell (dotted lines). **** denotes $\mathrm{P}<.0001$ when

213 compared to vector RNAi fed (Tukey's HSD). 


\section{Mianserin mimics DR by antagonizing the 5-HT1A receptor SER-4.}

215 Prior reports suggest that serotonin receptor orthologs ser-1 and ser-4 are necessary for the

216 lifespan benefits of mianserin in C. elegans $^{32}$, and we hypothesized that a subset of the serotonin

217 receptor orthologs will also be necessary for mianserin and DR-mediated fmo-2 induction. After

218 two generations of RNAi treatment, ser-1 and ser-4 were the only two receptors that proved

219 necessary for fmo-2 induction on mianserin (Figure 4A, Figure S7A-C) whereas ser-4

220 knockdown most robustly abrogated DR-mediated fmo-2 induction (Figure S7D-E). Further, we

221 see that ser-4(RNAi) slightly but significantly increases lifespan and prevents DR from extending

222 lifespan (Figure 4B), supporting the hypothesis that mianserin acts as a DR mimetic by

223 antagonizing serotonin signaling that occurs during feeding. Finally, to investigate whether this

224 effect is mediated by neuronal signaling or intestinal SER-4 expression, we rescued ser-4

225 knockout animals with tissue-specific promoters and found that only neuronal unc-119p::ser-4 is

226 sufficient to rescue full induction of fmo-2 under DR (Figure 4C-D). This is consistent with

227 serotonergic signaling within the nervous system, and not directly to the intestine, regulating the

228 response to food and food smell.

230 Thioridazine induces fmo-2 and extends lifespan through Dopamine receptor DOP-

\section{$231 \quad$ 3/DRD2.}

232 Thioridazine is a compound that antagonizes dopamine receptor D2 (DRD2) in mammals ${ }^{33}$, and

233 induces fmo-2 and mimics DR to increase longevity in nematodes (Figure 2). Based on its role

234 in mammals, we tested whether nematode DRD2 is involved in DR and mianserin-related fmo-2

235 induction and longevity. When the DRD2 ortholog dop-3 is knocked down by RNAi, fmo-2

236 induction is not affected in fed conditions but its induction by DR is diminished, while its 
237 induction by thioridazine is completely abrogated (Figure 4E, Figure S8A). This result is

238 consistent with $d o p-3$ being required for dopaminergic induction of fmo-2. To demonstrate the

239 epistasis of DOP-3 and SER-4 in the signaling pathway, we combined ser-4 RNAi with

240 mianserin and thioridazine treatment. The results show that ser-4 depletion blocks fmo-2

241 induction by thioridazine as well as suppresses fmo-2 induction by mianserin, as expected

242 (Figure 4A). Similarly, depletion of $d o p-3$ blocks both mianserin and thioridazine from inducing

243 fmo-2 (Figure 4E). These results support a model where both serotonin and dopamine signaling

244 are epistatic to each other and are each required for full induction of fmo-2 under DR.

245 Interestingly, when ser-4 or $d o p-3$ receptors are completely absent, via null mutation, the mutant

246 animals show dysregulation of fmo-2 induction, suggesting that the lack of biogenic amine

247 signaling increases variability in responding to environmental changes (Figure S7F-G, S8B-D).

248 To test whether DOP-3/DRD2 is necessary for lifespan extension by DR and mianserin, we

249 depleted dop-3 with RNAi under DR and found that dop-3 depletion increases lifespan but is not

250 further extended by DR (Figure 4F). Together, these results suggest that dopamine and serotonin

251 signaling interactively induce fmo-2 and extend lifespan under DR. 
A
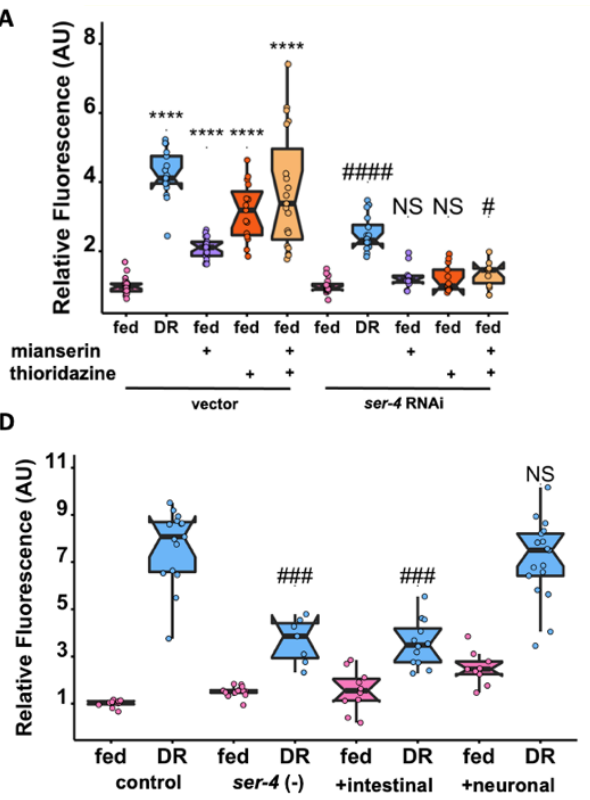

B

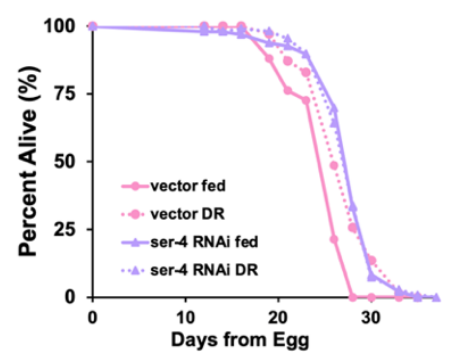

E

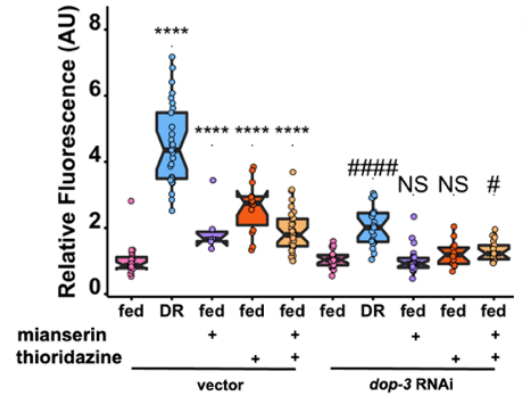

c

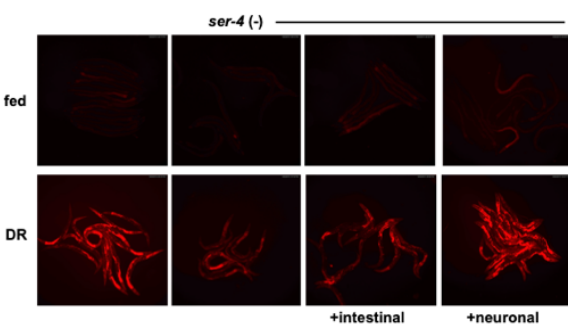

$\mathbf{F}$

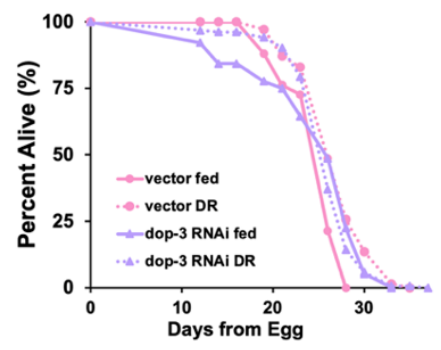

253 Figure 4. 5-HT1A receptor ser-4 and DRD2 receptor dop-3 act downstream of food perception.

254 Quantification (A) of individual fmo-2p::mCherry worms on fed (pink), and DR (blue) treated with

$255100 \mu \mathrm{M}$ mianserin (purple), $100 \mu \mathrm{M}$ thioridazine (orange), or combined (orange) worms fed vector or ser-

2564 RNAi. Survival curves $(\mathbf{B})$ of WT animals on vector RNAi in pink and ser-4 RNAi in purple on fed

257 (solid lines) or DR (dotted lines) conditions. Images $(\mathbf{C})$ and quantification $(\mathbf{D})$ of $f m o-2 p:: m C h e r r y$ or

258 ser-4 with tissue-specific rescues added back on fed (pink) and DR (blue). Quantification (E) of

259 individual fmo-2p::mCherry worms on fed (pink), and DR (blue) treated with $100 \mu \mathrm{M}$ mianserin (purple),

$260100 \mu \mathrm{M}$ thioridazine (orange), or combined (orange) worms fed vector or dop-3 RNAi. Survival curves

261 (F) of WT animals on vector RNAi in pink and dop-3 RNAi in purple on fed (solid lines) or DR (dotted

262 lines) conditions. **** denotes $\mathrm{P}<.0001$ when compared to vector RNAi fed (Tukey's HSD). \# denotes

$263 \mathrm{P}<.05$, \#\#\#\# denotes $\mathrm{P}<.0001$ when compared to ser-4/dop-3 RNAi fed (Tukey's HSD). 


\section{Mianserin induces FMOs and promotes stress resistance in mammalian cells.}

265 Having identified serotonin and dopamine antagonism upstream of fmo-2 induction under DR,

266 we were curious whether these relationships might be conserved. In mammals, previous studies

267 show interventions that increase longevity often both induced Fmo genes and increased stress

268 resistance ${ }^{13,34}$. Thus we tested whether mianserin and thioridazine are sufficient to induce

269 mammalian Fmo genes and whether this induction could confer stress resistance, as a surrogate

270 for longevity ${ }^{35}$. Our results, using human liver (HepG2) cells, show that while thioridazine did

271 not lead to any changes (Figure S9A), perhaps due to lack of DRD2 receptor expression,

272 mianserin treatment at $2 \mu \mathrm{M}$ increased protein levels of mammalian FMO2 (Figure 5A-B) and

273 FMO1 (Figure S9B-C), while $0.1 \mu \mathrm{M}$ mianserin increased protein levels of FMO4 (Figure S9B

274 and S9D). FMO3 and FMO5 protein levels are not changed upon mianserin treatment (Figure

275 S9B and S9E-F). Since stress resistance is often correlated with increased lifespan both within

276 and between species, and fmo-2 increases stress resistance in C. elegans, we next examined

277 whether mianserin also promotes stress resistance ${ }^{35}$. We treated cells with paraquat, an inducer

278 of mitochondrial oxidative stress through increased production of the reactive oxygen species

279 (ROS) superoxide, and find that $2 \mu \mathrm{M}$ mianserin, the dose that showed maximal induction of

280 FMOs, slightly but significantly improves the survival of HepG2 cells under an increasing dose

281 of paraquat (Figure S9G). These data support serotonin antagonism as a conserved mechanism to

282 induce Fmo expression and improve stress resistance. 


\section{Mianserin extends D. melanogaster lifespan similar to C. elegans.}

285 Since mianserin induces mammalian Fmos and promotes survival under paraquat stress, we

286 tested whether it also affects lifespan in evolutionarily distant species. Similar to data in worms,

287 recent data in the vinegar fly $D$. melanogaster show that altered serotonin signaling can change

288 their ability to assess caloric quality and modulate lifespan ${ }^{21}$. As we found a narrow range of

289 effective doses in worms (Figure 2F), we tested a slightly higher dose of mianserin in vinegar

290 flies (2 mM) for its effect on Fmo2 induction. The resulting data show that both mianserin and

291 fasting (DR) increase expression of fly fmo-2 expression (Figure 5C), but not fmo-1 (Figure

292 S10A). We then asked whether mianserin could also extend lifespan in flies. Using several

293 concentrations, we find a positive correlation between mianserin dosage and increased lifespan

294 until reaching a detrimental level of serotonin antagonism (Figure 5D, Figure S10B-D). We also

295 find a comparable dose response among male and female flies. We note that mianserin treatment

296 does not significantly alter food consumption (Figure S10E-F), as measured by the Fly Liquid-

297 Food Interaction Counter (FLIC) assay ${ }^{36}$. Together, these results are consistent with conserved

298 induction of fmos by mianserin and DR, in addition to conserved lifespan extension. 
A

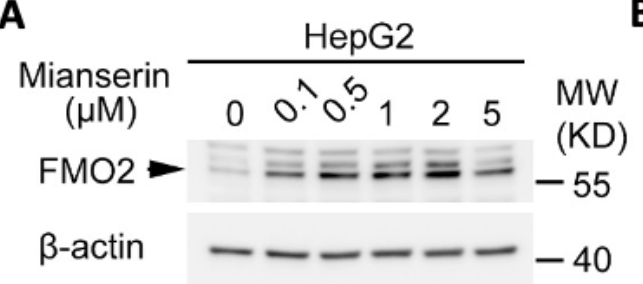

B

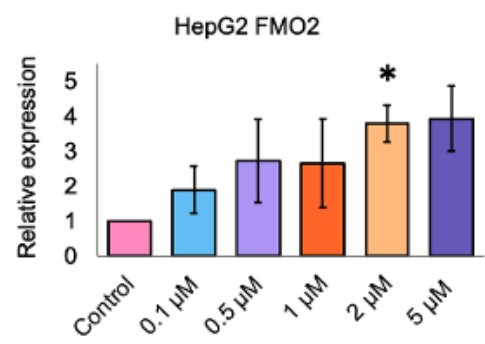

C

fmo-2 levels after 8 hours
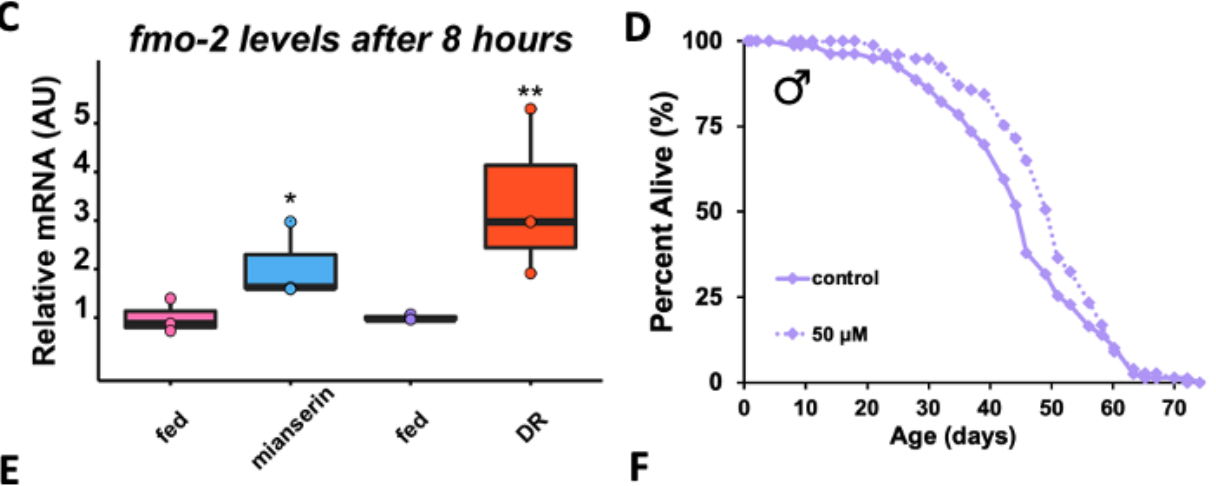

$\mathbf{F}$
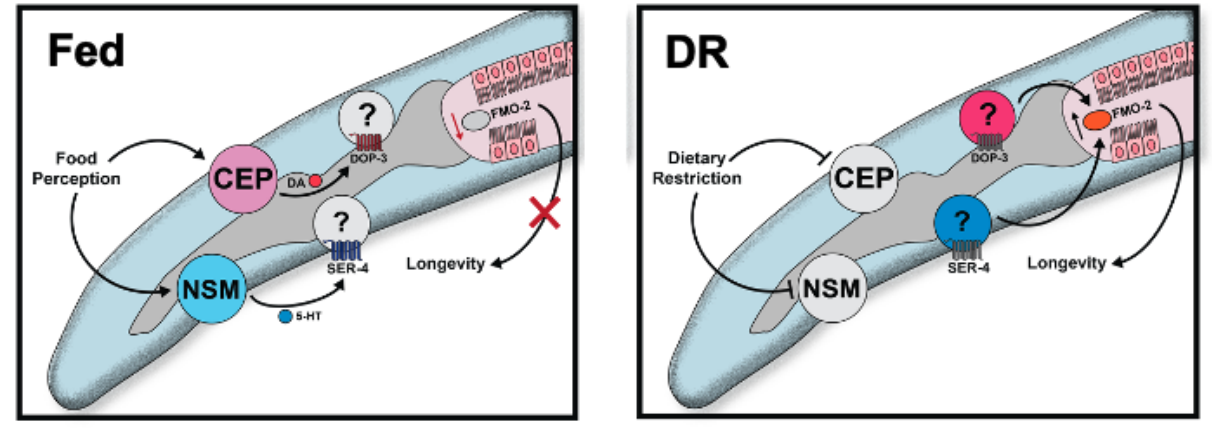

Figure 5. Serotonin antagonist mianserin induces FMO and improves health in Drosophila and

mammalian cells. Western blot image (A) and quantification (B) of FMO1 in whole cell lysates from HepG2 cells treated with $0.1 \mu \mathrm{M}, 0.5 \mu \mathrm{M}, 1 \mu \mathrm{M}, 2 \mu \mathrm{M}$, or $5 \mu \mathrm{M}$ mianserin. Fmo-2 mRNA levels (C) after eight hours of $2 \mathrm{mM}$ mianserin (blue) or starvation (orange) compared to water controls (pink and

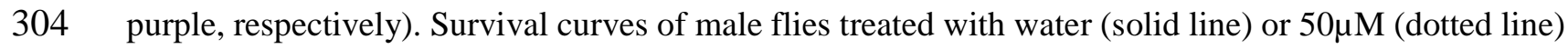

305 mianserin (D). Panels E and F depict the "on/off" state worm's toggle between when perceiving food.

* denotes $\mathrm{P}<.05, * *$ denotes $\mathrm{P}<.01$ when compared to control or fed (student's t-test). 


\section{Discussion}

308 Our experimental data in C. elegans support a model where the lack of an attractive (food) smell

309 leads to a loss of serotonin release from the enteric NSM neurons and lack of serotonin binding

310 to the SER-4/5-HT1A receptor. This in turn or in combination with other cues leads to a

311 reduction in dopamine signaling to downstream DOP-3/DRD2 receptors. It is notable that both

312 SER-4 and DOP-3 receptors are known to dampen adenylyl cyclase activity when bound, thus

313 the lack of signal will increase the probability of excitement of the cell expressing these

314 receptors. We hypothesize worms toggle their serotonin and dopamine neural activity "on" or

315 “off” depending on the presence or absence of food, respectively (Figure 5E-F). Based on our

316 ability to rescue DR benefits when food is perceived, we hypothesize that the perception of food

317 during DR prevents the benefits of DR, rather than shortening lifespan through an independent

318 pathway (Figure S10G). Critically, these data highlight that understanding how the nervous

319 system evaluates and appropriately integrates large amounts of external stimuli, like the

320 availability of food, allows us to target the decision-making processes to mimic pro-longevity

321 pathways.

323 It is intriguing that dopamine and serotonin signaling interactively induce fmo-2 and extend

324 lifespan in a common pathway induced by dietary restriction. In nematodes, slowing locomotion

325 in the presence of food is thought to be distinctly regulated by pharyngeal mechanosensation

326 leading to dopamine release while dwelling behavior is potentiated by serotonin ${ }^{37}$. Significant

327 scientific effort has identified much of the specific circuitry these neurotransmitters use to

328 promote changes in chemotaxis and egg-laying ${ }^{20,30,38-40}$. The results suggest worms can interpret

329 and implement a diverse set of responses to their changing environment. In mammals, SER-4/5- 
330 HT1A receptor activation increases dopamine release throughout the brain ${ }^{41,42}$. Similarly, recent

331 work shows release of serotonin and dopamine in the human brain influence non-reward-based

332 aspects of cognition and behavior like decision making ${ }^{43}$. These findings support a conserved

333 link between these two neurotransmitters in regulating complex phenotypes like aging.

335 It is also intriguing that one of these drugs, mianserin, successfully induces Fmo genes in both

336 mammals and flies, and leads to increased stress resistance and lifespan, respectively. Since

337 mianserin treatment extends fly lifespan we suspect it acts through a similar mechanism,

338 serotonin antagonism, to mimic DR. This hypothesis is bolstered by fmo-2 induction under acute

339 mianserin exposure and fasting, analogous to what we see in C. elegans. It is not known whether

340 FMOs or 5-HT1A receptors are necessary for mianserin or DR-mediated longevity in flies, but

3415 -HT2A receptors are necessary for proper food valuation ${ }^{21}$ suggesting that altering serotonin

342 signaling may prove fruitful in future studies. In cells, the induction of Fmos by mianserin must

343 be direct, suggesting that either serotonergic signaling is more direct in mammalian systems, or

344 more likely, there are other nuances in this signaling in mammals we do not yet understand.

345 Mammals and C. elegans share a single common ancestral Fmo ${ }^{15}$ and mammalian Fmos share

346 similar homology to C. elegans fmo-2, with Fmo5 having the highest \% identity. It is notable that

347 5-HT1A expression is detected in hepatocytes (The Human Protein Atlas), supporting a similar

348 mechanism in these cells and suggesting that FMOs can be induced by serotonin antagonism

349 both directly and indirectly. It will be interesting to investigate whether mianserin is beneficial

350 for health and longevity in mammals. To achieve this goal, it is imperative that we understand

351 the causative changes of pro-longevity drugs, such as atypical serotonin antagonists that are

352 known to have pleiotropic effects in humans. In addition to providing the potential for long-term 
353 health benefits, this knowledge will benefit our understanding of serotonin and dopamine

354 signaling networks that affect numerous human processes and diseases outside of aging.

356 Authorship Contributions

357 H.A.M., S.H., and S.F.L developed the conceptual framework and wrote the manuscript.

358 H.A.M., S.H., M.L.S., E.S.D., A.M.T., A.S.M., S.B., and S.F.L. contributed to data collection

359 and analysis. H.A.M. and E.S.D. prepared the figures and tables. All authors reviewed and

360 approved the manuscript.

\section{References}

363 1. López-Otín C, Blasco MA, Partridge L, Serrano M, Kroemer G. The hallmarks of aging. Cell. Jun 2013;153(6):1194-217. doi:10.1016/j.cell.2013.05.039

3652 2. Medkour Y, Svistkova V, Titorenko VI. Cell-Nonautonomous Mechanisms Underlying Cellular and Organismal Aging. Int Rev Cell Mol Biol. 2016;321:259-97.

doi.10.1016/bs.ircmb.2015.09.003 and longevity. Elife. Dec 2020;9doi:10.7554/eLife.62659 4. Berger M, Gray JA, Roth BL. The expanded biology of serotonin. Annu Rev Med. 2009;60:355-66. doi:10.1146/annurev.med.60.042307.110802 Dopaminergic reward system: a short integrative review. Int Arch Med. Oct 2010;3:24. doi:10.1186/1755-7682-3-24 6. Tatum MC, Ooi FK, Chikka MR, et al. Neuronal serotonin release triggers the heat shock response in C. elegans in the absence of temperature increase. Curr Biol. Jan 2015;25(2):163-74. doi:10.1016/j.cub.2014.11.040

378 7. Leiser SF, Miller H, Rossner R, et al. Cell nonautonomous activation of flavin-containing monooxygenase promotes longevity and health span. Science. Nov

$380 \quad$ 2015;doi:10.1126/science.aac9257

381 8. Grady DL, Thanos PK, Corrada MM, et al. DRD4 genotype predicts longevity in mouse 382 and human. J Neurosci. Jan 2013;33(1):286-91. doi:10.1523/JNEUROSCI.3515-12.2013

383 9. $\quad$ Saharia K, Kumar R, Gupta K, Mishra S, Subramaniam JR. Reserpine requires the D2384 type receptor,. J Biosci. Dec 2016;41(4):689-695. doi:10.1007/s12038-016-9652-7

385 10. Yin JA, Liu XJ, Yuan J, Jiang J, Cai SQ. Longevity manipulations differentially affect serotonin/dopamine level and behavioral deterioration in aging Caenorhabditis elegans. $J$

388 11. Peters R. Ageing and the brain. Postgrad Med J. Feb 2006;82(964):84-8.

389 doi:10.1136/pgmj.2005.036665 
390 12. Fontana L, Partridge L, Longo VD. Extending Healthy Life Span--From Yeast to Humans. Science. April 16, 2010 2010;328(5976):321-326. doi:10.1126/science.1172539 13. Steinbaugh MJ, Sun LY, Bartke A, Miller RA. Activation of genes involved in xenobiotic metabolism is a shared signature of mouse models with extended lifespan. Am J Physiol Endocrinol Metab. Aug 2012;303(4):E488-95. doi:10.1152/ajpendo.00110.2012 14. Swindell WR. Genes and gene expression modules associated with caloric restriction and aging in the laboratory mouse. BMC Genomics. 2009;10:585. doi:10.1186/1471-2164-10-585 15. Petalcorin MI, Joshua GW, Agapow PM, Dolphin CT. The fmo genes of Caenorhabditis elegans and C. briggsae: characterisation, gene expression and comparative genomic analysis. Gene. Feb 2005;346:83-96. doi:10.1016/j.gene.2004.09.021 16. Kaeberlein TL, Smith ED, Tsuchiya M, et al. Lifespan extension in Caenorhabditis elegans by complete removal of food. Aging Cell. Dec 2006;5(6):487-94.

403

404 17. Bargmann CI, Hartwieg E, Horvitz HR. Odorant-selective genes and neurons mediate olfaction in C. elegans. Cell. Aug 1993;74(3):515-27. doi:10.1016/0092-8674(93)80053-h 18. Worthy SE, Haynes L, Chambers M, et al. Identification of attractive odorants released by preferred bacterial food found in the natural habitats of C. elegans. PLoS One. 2018;13(7):e0201158. doi:10.1371/journal.pone.0201158 19. Nuttley WM, Atkinson-Leadbeater KP, Van Der Kooy D. Serotonin mediates food-odor associative learning in the nematode Caenorhabditiselegans. Proc Natl Acad Sci U S A. Sep 2002;99(19):12449-54. doi:10.1073/pnas.192101699

20. Flavell SW, Pokala N, Macosko EZ, Albrecht DR, Larsch J, Bargmann CI. Serotonin and the neuropeptide PDF initiate and extend opposing behavioral states in C. elegans. Cell. Aug 2013;154(5):1023-35. doi:10.1016/j.cell.2013.08.001

21. Ro J, Pak G, Malec PA, et al. Serotonin signaling mediates protein valuation and aging. Elife. Aug 2016;5doi:10.7554/eLife.16843

22. Linford NJ, Ro J, Chung BY, Pletcher SD. Gustatory and metabolic perception of nutrient stress in Drosophila. Proc Natl Acad Sci U S A. Feb 2015;112(8):2587-92. doi:10.1073/pnas.1401501112

23. Marshall RJ. The pharmacology of mianserin--an update. Br J Clin Pharmacol. 1983;15 Suppl 2:263S-268S. doi:10.1111/j.1365-2125.1983.tb05874.x

\section{Shana M. Feinberg KF, Abdolreza Saadabadi. StatPearls. Thioridazine. Stat Pearls;} 2020.

25. Petrascheck M, Ye X, Buck LB. An antidepressant that extends lifespan in adult Caenorhabditis elegans. Nature. Nov 2007;450(7169):553-6. doi:10.1038/nature05991 26. Bishop NA, Guarente L. Two neurons mediate diet-restriction-induced longevity in C. elegans. Nature. May 2007;447(7144):545-9. doi:10.1038/nature05904

27. Murakami H, Murakami S. Serotonin receptors antagonistically modulate Caenorhabditis elegans longevity. Aging Cell. Aug 2007;6(4):483-8. doi:10.1111/j.1474-9726.2007.00303.x

28. Sze JY, Victor M, Loer C, Shi Y, Ruvkun G. Food and metabolic signalling defects in a Caenorhabditis elegans serotonin-synthesis mutant. Nature. Feb 2000;403(6769):560-4. doi: $10.1038 / 35000609$

29. Trent C, Tsung N, Horvitz HR. Egg-laying defective mutants of the nematode Caeenorhabditis Elegans. Genetics. August 1, 1983 1983;104(4):619-647.

30. Rhoades JL, Nelson JC, Nwabudike I, et al. ASICs Mediate Food Responses in an

435 97.e14. doi:10.1016/j.cell.2018.11.023 
31. Churgin MA, McCloskey RJ, Peters E, Fang-Yen C. Antagonistic Serotonergic and Octopaminergic Neural Circuits Mediate Food-Dependent Locomotory Behavior in Caenorhabditis elegans. J Neurosci. Aug 2017;37(33):7811-7823. doi:10.1523/JNEUROSCI.2636-16.2017 32. Petrascheck M, Ye X, Buck LB. A high-throughput screen for chemicals that increase the lifespan of Caenorhabditis elegans. Ann N Y Acad Sci. Jul 2009;1170:698-701. doi:10.1111/j.1749-6632.2009.04377.x 33. StatPearls. 2020.

34. Murakami S, Salmon A, Miller RA. Multiplex stress resistance in cells from long-lived dwarf mice. FASEB J. Aug 2003;17(11):1565-6. doi:10.1096/fj.02-1092fje

\section{Harper JM, Salmon AB, Leiser SF, Galecki AT, Miller RA. Skin-derived fibroblasts} from long-lived species are resistant to some, but not all, lethal stresses and to the mitochondrial inhibitor rotenone. Aging Cell. Feb 2007;6(1):1-13. doi:ACE255 [pii] 10.1111/j.1474-9726.2006.00255.x

36. Ro J, Harvanek ZM, Pletcher SD. FLIC: high-throughput, continuous analysis of feeding behaviors in Drosophila. PLoS One. 2014;9(6):e101107. doi:10.1371/journal.pone.0101107

452 37. Sawin ER, Ranganathan R, Horvitz HR. C. elegans locomotory rate is modulated by the environment through a dopaminergic pathway and by experience through a serotonergic pathway. Neuron. Jun 2000;26(3):619-31. doi:10.1016/s0896-6273(00)81199-x

38. Cermak N, Yu SK, Clark R, Huang YC, Baskoylu SN, Flavell SW. Whole-organism behavioral profiling reveals a role for dopamine in state-dependent motor program coupling in. Elife. Jun 2020;9doi:10.7554/eLife.57093 39. Hills T, Brockie PJ, Maricq AV. Dopamine and glutamate control area-restricted search behavior in Caenorhabditis elegans. J Neurosci. Feb 2004;24(5):1217-25. doi:10.1523/JNEUROSCI.1569-03.2004

40. Lee KS, Iwanir S, Kopito RB, et al. Serotonin-dependent kinetics of feeding bursts underlie a graded response to food availability in C. elegans. Nat Commun. 02 2017;8:14221. doi:10.1038/ncomms14221

41. Li Z, Ichikawa J, Dai J, Meltzer HY. Aripiprazole, a novel antipsychotic drug, preferentially increases dopamine release in the prefrontal cortex and hippocampus in rat brain. Eur J Pharmacol. Jun 2004;493(1-3):75-83. doi:10.1016/j.ejphar.2004.04.028

42. Bantick RA, De Vries MH, Grasby PM. The effect of a 5-HT1A receptor agonist on striatal dopamine release. Synapse. Aug 2005;57(2):67-75. doi:10.1002/syn.20156

43. Bang D, Kishida KT, Lohrenz T, et al. Sub-second Dopamine and Serotonin Signaling in Human Striatum during Perceptual Decision-Making. Neuron. Oct

472 2020;doi:10.1016/j.neuron.2020.09.015 